DOI: https://doi.org/10.24297/jam.v17i0.8336

\title{
Convergence of the Collatz Sequence
}

\author{
Anatoliy Nikolaychuk \\ 855 N Vermont Ave, Los Angeles, CA 90029, Los Angeles City College \\ Tanya41tolya41@yahoo.com
}

\section{Abstract}

For any natural number was created the supplement sequence, that is convergent together with the original Collatz sequence. The numerical parameter - index was defined, that is the same for both sequences. This new method provides the following results:

1. All natural numbers were distributed into six different classes;

2. The properties of the index were found for the different classes;

3. For any natural number was constructed the bounded sequence of increasing numbers, that is convergent together with the regular Collatz sequence.

Keywords: Collatz Sequence

\section{Introduction}

For any natural number the Collatz sequence defined by the rule (Ref.1):

$a_{n+1}=\left\{\begin{array}{l}\frac{1}{2} a_{n}, \text { if } a_{n} \text { is even } \\ 3 a_{n}+1, \text { if } a_{n} \text { is odd }\end{array}\right.$

Thus, for $\mathrm{N}=11$, the corresponding sequence is

$11,34,17,52,26,13,40,20,10,5,16,8,4,2,1$, and the sequence will repeat itself. The number $N$ is convergent if the corresponding sequence contains 1 .

For the natural number $\mathrm{N}=2^{p} \cdot(2 k+1) \quad(p=0,1,2,3, \ldots ; k=0,1,2,3, \ldots)$ were defined as the index $\delta(\mathrm{N})$ by the following (Ref.2):

1. $\delta(1)=0$

2. $\delta(\mathrm{N})=\left\{\begin{array}{l}\delta(3 \mathrm{~N}+1)+1, \text { if } \mathrm{N} \text { is odd } \\ \delta\left(\frac{1}{2} \mathrm{~N}\right)+1, \quad \text { if } \mathrm{N} \text { is even }\end{array}\right.$

For example, if $\mathrm{N}=11, \delta(11)=14$, which is the number of steps number 11 goes to 1 .

Materials and Methods: Abstract proofs 


\section{Results and Discussion:}

1. All natural numbers were distributed into six different classes;

2. The properties of the index were found for the different classes;

3. For any natural number was constructed the bounded sequence of increasing numbers, that is convergent together with the regular Collatz sequence.

\section{Main Text:}

The Supplement Sequences.

For the original sequence corresponding to number $\mathrm{N}$, we may write different supplement sequences, that are convergent together with the original sequence, and all such sequences have the same index.

Example. For the sequence corresponding to $\mathrm{N}=26$, we may construct another sequence by the rule:

$$
a_{n+1}= \begin{cases}\frac{a_{n}-1}{2} & \text { if } a_{n} \text { is odd } \\ a_{n} \cdot \frac{3}{2}+1 & \text { if } a_{n} \text { is even }\end{cases}
$$

and we received two "relative" sequences:

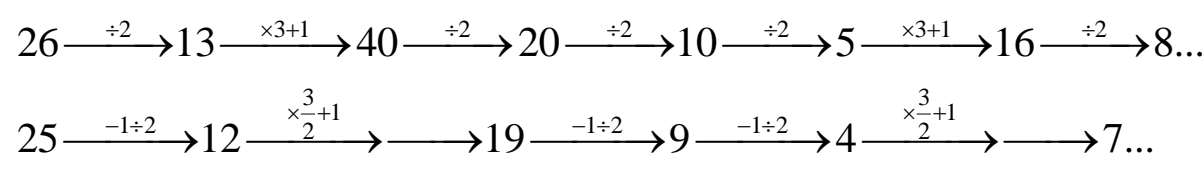

or we may construct other sequences

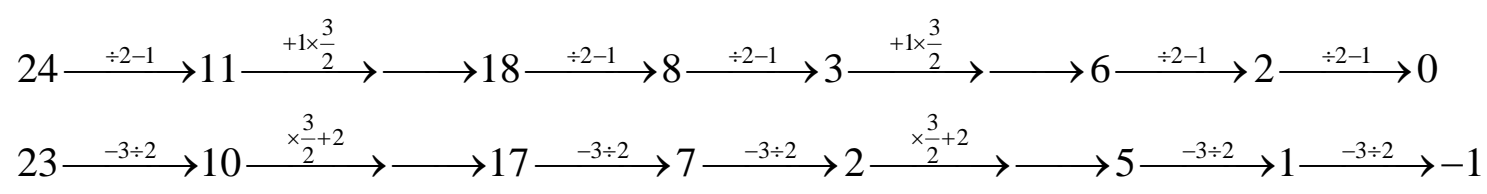

The reader may construct different supplement sequences, by finding an appropriate rule, such that all those sequences are convergent with the same index.

Basic Supplement Sequence.

In this article, we will consider the supplement sequence defined by the rule (Ref.2):

$a_{n+1}=\left\{\begin{array}{l}\frac{a_{n}+1}{2} \text { if } a_{n} \text { is odd } \\ a_{n} \cdot \frac{3}{2} \quad \text { if } a_{n} \text { is even }\end{array}\right.$

Example: For the number 26 the original and supplement sequences can be written 


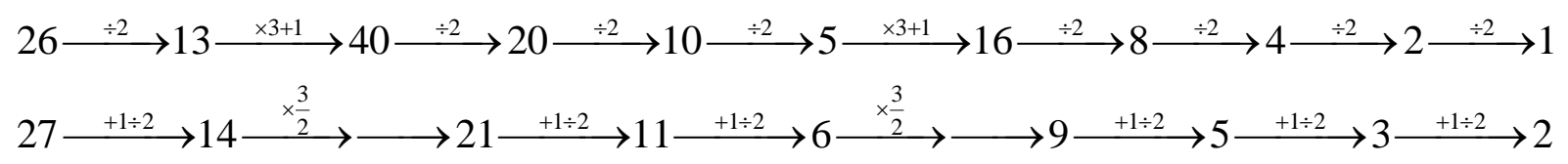

We see that numbers in the second raw exceed corresponding numbers in the first raw by one. For the new supplement sequence we define the operations:

$A\left(a_{n}\right)=\frac{a_{n}+1}{2}$ if $a_{n}$ is odd and $B\left(a_{n}\right)=a_{n} \cdot \frac{3}{2}$ if $a_{n}$ is even,

and inverse operations $A^{-1}\left(a_{n}\right)=a_{n} \cdot 2-1, B^{-1}\left(a_{n}\right)=a_{n} \cdot \frac{2}{3}$.

Theorem 3.1 If the natural number $\mathrm{N}$ converges to 1 with index $\delta$ (the number of steps in the original sequence), then the corresponding supplement sequence for the number $N+1$ converges to 2 with the same index, where the operation $A$ is equivalent to one step, and the operation B is equivalent to two steps. Numbers in supplement sequence exceed corresponding numbers in original sequence exactly by 1 .

Proof. a) If $a_{n}$ is odd, then in the original sequence $a_{n} \stackrel{\times 3+1}{\longrightarrow} 3 \cdot a_{n}+1 \stackrel{\div 2}{\longrightarrow} \frac{3 a_{n}+1}{2}$.

For the supplement sequence, $a_{n}+1$ is even, and $a_{n}+1 \stackrel{B}{\longrightarrow} \frac{\left(a_{n}+1\right) \cdot 3}{2}=\frac{3 \cdot a_{n}+1}{2}+1$.

b) If $a_{n}$ is even, then in the original sequence $a_{n} \stackrel{\div 2}{\longrightarrow} \frac{a_{n}}{2}$, and the corresponding supplement sequence $a_{n}+1 \stackrel{A}{\longrightarrow} \frac{a_{n}+1+1}{2}=\frac{a_{n}}{2}+1$.

Corollary. The operation A reduces the index of a number by 1 and the operation $B$ reduces index by 2 , whereas inverse operations $A^{-1}$ and $B^{-1}$ increase the index by 1 and by 2 correspondingly.

Theorem 3.2. For the even number $\mathrm{N}=4 \mathrm{k}+2(\mathrm{k}=0,1,2,3, \ldots), \quad \delta(4 k+2)=\delta(3 k+2)+3$.

Proof. $4 k+2 \stackrel{B}{\longrightarrow} 6 k+3 \stackrel{A}{\longrightarrow} 3 k+2$, and both operations reduce index totally by 3 .

Corollary. From Theorem 3.2 we see that the even number $4 k+2$ is possible to reduce to the smaller number $3 k+2$. Any odd number can be reduced in one step using operation $A$. Therefore, to prove that any natural number converges, it is enough to show this only for the numbers which are divisible by 4 .

Properties of the index.

We separate all natural numbers by the six groups: 
Group $1 \quad N=2^{n}(4 k+1), \mathrm{n}$ is odd, $\mathrm{n} \geq 3, \mathrm{k}=0,1,2, \ldots$

and $N=2^{n}(4 k+3), \mathrm{n}$ is even, $\mathrm{n} \geq 2, \mathrm{k}=0,1,2, \ldots$;

Group 2 $N=2(4 k+1), \mathrm{k}=0,1,2, \ldots ;$

Group $3 \quad N=4 k+3, \mathrm{k}=0,1,2, \ldots ;$

Group 4 $N=2^{n}(4 k+1), \mathrm{n}$ is even, $\mathrm{n} \geq 2$ ，

and $N=2^{n}(4 k+3), \mathrm{n}$ is odd, $\mathrm{n} \geq 3, \mathrm{k}=0,1,2, \ldots$;

Group $5 \quad N=2(4 k+3), \mathrm{k}=0,1,2, \ldots$

Group $6 \quad N=4 k+1, \mathrm{k}=0,1,2, \ldots$

Lemma 4.1. $\frac{3^{n}+1}{2}\left\{\begin{array}{l}\text { is even, if } \mathrm{n} \text { is odd } \\ \text { is odd, if } \mathrm{n} \text { is even }\end{array}\right.$

We recognize that because $3^{n}+1$ is divisible by 4 if $\mathrm{n}$ is odd, and $3^{n}+1$ is divisible by 2 but not by 4 if $\mathrm{n}$ is even.

Theorem 4.1. If $\mathrm{n}$ is even, $n \geq 2$, then for the numbers from group 4 ,

$$
\delta\left[2^{n}(4 k+1)\right]=\delta\left[2^{n-1}(4 k+1)\right]+1 .
$$

Proof. Using lemma 4.1, we obtain

$$
\begin{aligned}
& 2^{n}(4 k+1) \stackrel{B^{n}}{\longrightarrow} 3^{n}(4 k+1) \stackrel{A}{\longrightarrow} 3^{n} \cdot 2 k+\frac{3^{n}+1}{2} \stackrel{A}{\longrightarrow} 3^{n} \cdot k+\frac{3^{n}+3}{4} ; \\
& 2^{n-1}(4 k+1) \stackrel{B^{n-1}}{\longrightarrow} 3^{n-1}(4 k+1) \stackrel{A}{\longrightarrow} 3^{n-1} \cdot 2 k+\frac{3^{n-1}+1}{2} \stackrel{B}{\longrightarrow} 3^{n} \cdot k+\frac{3^{n}+3}{4} .
\end{aligned}
$$

We see that $\delta\left[2^{n}(4 k+1)\right]=\delta\left[3^{n} \cdot k+\frac{3^{n}+3}{4}\right]+(n+2)$, and

$\delta\left[2^{n-1}(4 k+1)\right]=\delta\left[3^{n} \cdot k+\frac{3^{n}+3}{4}\right]+(n+1)$, which gives the proof of the Theorem 4.1.

Theorem 4.1 shows that numbers from group 4 are possible to reduce to twice smaller numbers with an index lower by one. Table 4.1 gives a list of such "good numbers." 


\begin{tabular}{|l|l|l|l|l|l|l|l|l|l|l|l|l|}
\hline \multicolumn{1}{|l|}{$\mathrm{k}$} & 0 & 1 & 2 & 3 & 4 & 5 & 6 & 7 & 8 & 9 & 10 & $\ldots$ \\
\hline 2 & 4 & 20 & 36 & 52 & 68 & 84 & 100 & 116 & 132 & 148 & 164 & $\ldots$ \\
\hline 4 & 16 & 80 & 144 & 208 & 272 & 336 & 400 & 464 & 528 & 592 & 656 & $\ldots$ \\
\hline 6 & 64 & 320 & 576 & 832 & 1088 & 1344 & 1600 & 1856 & 2112 & 2368 & 2624 & $\ldots$ \\
\hline 8 & 256 & 1280 & 2304 & 3328 & 4352 & 5376 & 6400 & 7424 & 8448 & 9472 & 10,496 & $\ldots$ \\
\hline$\ldots$ & & & & & & & & & & & & \\
\hline
\end{tabular}

Theorem 4.2. If $n$ is odd $(n=1,3,5, \ldots)$, then for the numbers from group 4 ,

$\delta\left[2^{n}(4 k+3)\right]=\delta\left[2^{n-1}(4 k+3)\right]+1$.

Proof. $2^{n}(4 k+3) \stackrel{B^{n}}{\longrightarrow} 3^{n}(4 k+3) \stackrel{A}{\longrightarrow} 3^{n} \cdot 2 k+\frac{3^{n+1}+1}{2} \stackrel{A}{\longrightarrow} 3^{n} \cdot k+\frac{3^{n+1}+3}{4}$;

$2^{n-1}(4 k+3) \stackrel{B^{n-1}}{\longrightarrow} 3^{n-1}(4 k+3) \stackrel{A}{\longrightarrow} 3^{n-1} \cdot 2 k+\frac{3^{n}+1}{2} \stackrel{B}{\longrightarrow} 3^{n} \cdot k+\frac{3^{n+1}+3}{4}$.

The last entries are the same, and $\delta\left[2^{n}(4 k+3)\right]=\delta\left[3^{n} \cdot k+\frac{3^{n+1}+3}{4}\right]+(n+2)$, whereas

$\delta\left[2^{n-1}(4 k+3)\right]=\delta\left[3^{n} \cdot k+\frac{3^{n+1}+3}{4}\right]+(n+1)$, and we get a proof of Theorem 4.2.

Table 4.2 gives some numbers from group 4 that is possible to reduce to twice lower numbers

\begin{tabular}{|l|l|l|l|l|l|l|l|l|l|l|l|l|}
\hline \multicolumn{1}{|c|}{$\mathrm{k}$} & 0 & 1 & 2 & 3 & 4 & 5 & 6 & 7 & 8 & 9 & 10 & $\ldots$ \\
\hline 3 & 24 & 56 & 88 & 120 & 152 & 184 & 216 & 248 & 280 & 312 & 344 & $\ldots$ \\
\hline 5 & 96 & 224 & 352 & 480 & 608 & 736 & 864 & 992 & 1120 & 1248 & 1376 & $\ldots$ \\
\hline 7 & 384 & 896 & 1408 & 1920 & 2432 & 2944 & 3456 & 3968 & 4480 & 4992 & 5504 & $\ldots$ \\
\hline$\ldots$ & & & & & & & & & & & & \\
\hline
\end{tabular}

Theorem 4.3 For the numbers from groups 1, 2, and 3, $\delta(N)=\delta(3 N)+1$. 


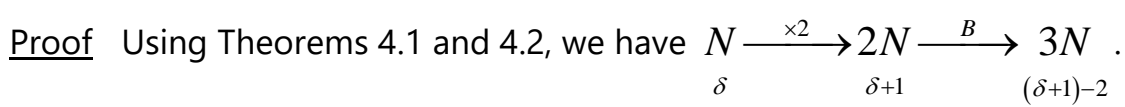

Theorem 4.4 If $\mathrm{N}$ is even, then $\delta(N)=\delta(3 N-1)+1$.

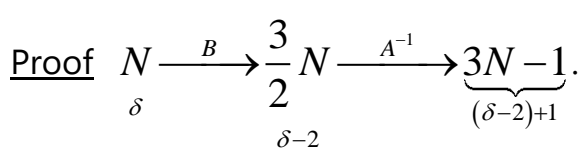

Example 4.1

\begin{tabular}{|l|l|l|l|l|l|l|}
\hline & Group 1 & index & Group 2 & index & Group 3 & index \\
\hline $\mathrm{N}$ & 240 & 52 & 178 & 31 & 359 & 32 \\
\hline $3 \mathrm{~N}$ & 720 & 51 & 534 & 30 & 1077 & 31 \\
\hline $3 \mathrm{~N}-1$ & 719 & 51 & 533 & 30 & 1076 & 31 \\
\hline
\end{tabular}

Theorem 4.5 For the numbers from groups 1, 2, and 3, $\delta(N)=\delta(4 N-1)-2$.

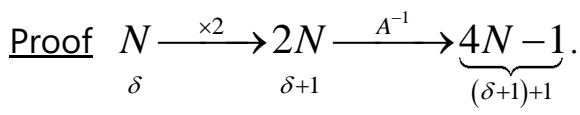

Theorem 4.6 If $\mathrm{N}$ is even, then $\delta(N)=\delta(4 N-2)-2$.

Proof $N \underset{\delta}{\stackrel{A^{-1}}{\longrightarrow}} \underbrace{2 N-1}_{\delta+1} \stackrel{\times 2}{\longrightarrow} \underbrace{4 N-2}_{(\delta+1)+1}$.

Theorem 4.7 For any natural number $\mathrm{N}, \delta(N)=\delta(4 N-3)-2$.

Proof $N \underset{\delta}{\stackrel{A^{-1}}{\longrightarrow}} \underbrace{2 N-1}_{\delta+1} \stackrel{A^{-1}}{\longrightarrow} \underbrace{4 N-3}_{(\delta+1)+1}$.

Example 4.2

\begin{tabular}{|l|l|l|l|l|l|l|}
\hline & Group 3 & index & Group 5 & index & Group 6 & index \\
\hline $\mathrm{N}$ & 295 & 117 & 182 & 18 & 357 & 32 \\
\hline $4 \mathrm{~N}-1$ & 1179 & 119 & & & & \\
\hline $4 \mathrm{~N}-2$ & & & 726 & 20 & & \\
\hline $4 \mathrm{~N}-3$ & 1177 & 119 & 725 & 20 & 1425 & 34 \\
\hline
\end{tabular}


Theorem 4.8 For the numbers from groups 1,2 , and $3, \delta(N)=\delta(6 N-1)$.

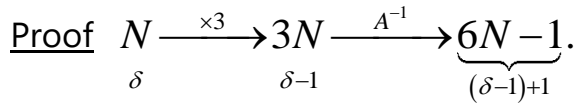

Theorem 4.9 If $\mathrm{N}$ is divisible by 4 , then $\delta(N)=\delta(6 N-2)$.

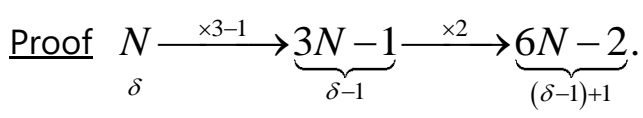

Theorem 4.10 If $\mathrm{N}$ is even, then $\delta(N)=\delta(6 N-3)$.

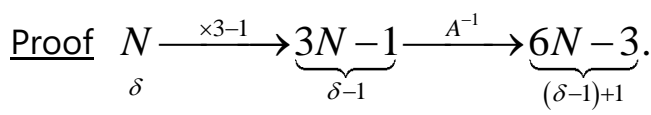

Example 4.3

\begin{tabular}{|l|l|l|l|l|l|l|}
\hline & Group 2 & index & Group 4 & index & Group 5 & index \\
\hline $\mathrm{N}$ & 146 & 116 & 272 & 42 & 150 & 23 \\
\hline $6 \mathrm{~N}-1$ & 875 & 116 & & & & \\
\hline $6 \mathrm{~N}-2$ & & & 1630 & 42 & & \\
\hline $6 \mathrm{~N}-3$ & 873 & 116 & 1629 & 42 & 897 & 23 \\
\hline
\end{tabular}

Theorem 4.11 For the numbers from groups 1,2 , and $3, \delta(N)=\delta(8 N-2)-3=\delta(8 N-3)-3$.

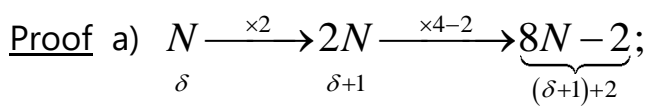

b) $N \stackrel{\times 2}{\longrightarrow} 2 N \stackrel{\times 4-3}{\longrightarrow} \underbrace{8 N-3}_{\delta+1}$.

Theorem 4.12 If $\mathrm{N}$ is even, then $\delta(N)=\delta(8 N-5)-3$.

Proof $N \stackrel{\times 4-2}{\longrightarrow} \underbrace{4 N-2}_{\delta+2} \stackrel{A^{-1}}{\longrightarrow} \underbrace{8 N-5}_{(\delta+2)+1}$.

Example 4.4

\begin{tabular}{|l|l|l|l|l|l|l|}
\hline & Group 2 & index & Group 3 & index & Group 5 & index \\
\hline $\mathrm{N}$ & 202 & 18 & 323 & 99 & 286 & 104 \\
\hline
\end{tabular}




\begin{tabular}{|l|l|l|l|l|l|l|}
\hline $8 \mathrm{~N}-2$ & 1614 & 21 & 2582 & 102 & & \\
\hline $8 \mathrm{~N}-3$ & 1613 & 21 & 2581 & 102 & & \\
\hline $8 \mathrm{~N}-5$ & 1611 & 21 & & & 2283 & 107 \\
\hline
\end{tabular}

Theorem 4.13 For the numbers from groups 1, 2, and 3, $\delta(N)=\delta(9 N-1)+2$.

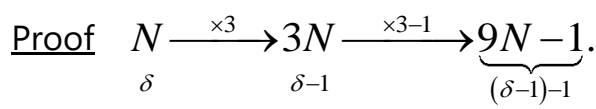

Theorem 4.14 If $\mathrm{N}$ is divisible by 4 , then $\delta(N)=\delta(9 N-3)+2$.

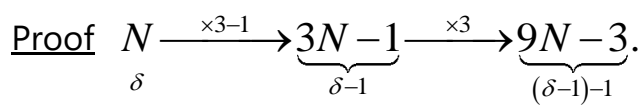

Example 4.5

\begin{tabular}{|l|l|l|l|l|l|l|}
\hline & Group 1 & index & Group 2 & index & Group 4 & index \\
\hline $\mathrm{N}$ & 368 & 45 & 282 & 42 & 612 & 38 \\
\hline $9 \mathrm{~N}-1$ & 3311 & 43 & 2537 & 40 & & \\
\hline $9 \mathrm{~N}-3$ & 3309 & 43 & & & 5505 & 36 \\
\hline
\end{tabular}

Theorem 4.15 For the numbers from groups 1 and $2, \delta(N)=\delta(12 N-2)-1$.

Proof $N \underset{\delta}{N} \underset{\delta-1}{\longrightarrow} \underset{\stackrel{\times 4-2}{\longrightarrow}}{\longrightarrow} \underbrace{12 N-2}_{(\delta-1)+2}$.

Theorem 4.16 For the numbers from groups 1,2 , and $3, \delta(N)=\delta(12 N-3)-1$.

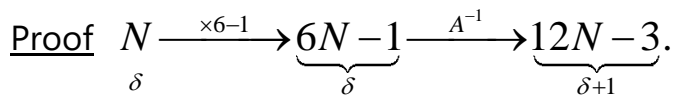

Theorem 4.17 If $\mathrm{N}$ is divisible by 4 , then $\delta(N)=\delta(12 N-5)-1$.

Proof $\quad N \stackrel{\times 6-2}{\longrightarrow} \underbrace{6 N-2}_{\delta} \stackrel{A^{-1}}{\longrightarrow} \underbrace{12 N-5}_{\delta+1}$.

Theorem 4.18 If $\mathrm{N}$ is even, then $\delta(N)=\delta(12 N-7)-1$.

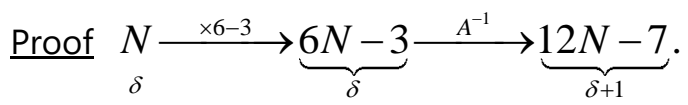




\section{Example 4.6}

\begin{tabular}{|l|l|l|l|l|l|l|}
\hline & Group 2 & index & Group 3 & index & Group 5 & index \\
\hline $\mathrm{N}$ & 882 & 116 & 419 & 40 & 326 & 24 \\
\hline $12 \mathrm{~N}-2$ & 10582 & 117 & & & & \\
\hline $12 \mathrm{~N}-3$ & 10581 & 117 & 5025 & 41 & & \\
\hline $12 \mathrm{~N}-7$ & 10577 & 117 & & & 3905 & 25 \\
\hline
\end{tabular}

Theorem 4.19 For the numbers from group $1, \delta(N)=\delta(18 N-2)+1=\delta(27 N-3)+3$.

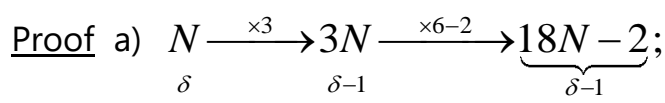

b) $\underset{\delta}{N \underset{3}{\longrightarrow}} 3 N \stackrel{\times 9-3}{\longrightarrow} \underbrace{27 N-3}_{(\delta-1)-2}$.

Theorem 4.20 For the numbers from groups 1 and 2, $\delta(N)=\delta(18 N-3)+1$.

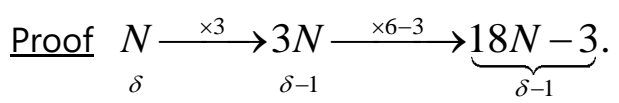

Theorem 4.21 If $\mathrm{N}$ is divisible by 4 , then $\delta(N)=\delta(18 N-7)+1$.

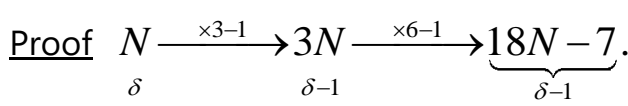

Example 4.7

\begin{tabular}{|l|l|l|l|l|l|l|}
\hline & Group 1 & index & Group 1 & index & Group 2 & index \\
\hline $\mathrm{N}$ & 296 & 55 & 460 & 128 & 306 & 37 \\
\hline $18 \mathrm{~N}-2$ & 5326 & 54 & 8278 & 127 & & \\
\hline $18 \mathrm{~N}-3$ & 5325 & 54 & 8277 & 127 & 5505 & 36 \\
\hline $18 \mathrm{~N}-7$ & 5321 & 54 & 8273 & 127 & & \\
\hline $27 \mathrm{~N}-3$ & 7989 & 52 & 12,420 & 125 & & \\
\hline
\end{tabular}

Theorem 4.22 For the numbers from groups 1,2, and 3,

$$
\delta(N)=\delta(16 N-5)-4=\delta(16 N-7)-4
$$




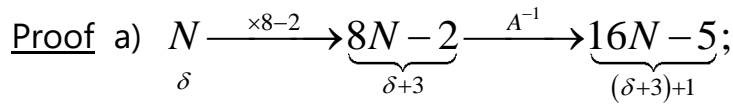

b) $\quad N \stackrel{\times 8-3}{\longrightarrow} \underbrace{8 N-3}_{\delta+3} \stackrel{A^{-1}}{\longrightarrow} \underbrace{16 N-7}_{(\delta+3)+1}$.

Theorem 4.23 If $\mathrm{N}$ is even, then $\delta(N)=\delta(16 N-10)-4=\delta(16 N-11)-4$.

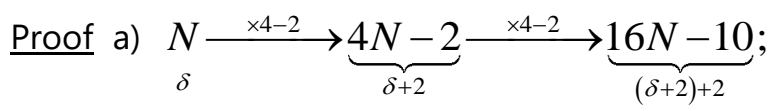

b) $N \stackrel{\times 4-2}{\longrightarrow} \underbrace{4 N-2}_{\delta+2} \stackrel{\times 4-3}{\longrightarrow} \underbrace{16 N-11}_{(\delta+2)+2}$.

Example 4.8

\begin{tabular}{|l|l|l|l|l|l|l|}
\hline & Group 3 & index & Group 4 & index & Group 5 & index \\
\hline $\mathrm{N}$ & 623 & 87 & 472 & 128 & 614 & 38 \\
\hline $16 \mathrm{~N}-5$ & 9963 & 91 & & & & \\
\hline $16 \mathrm{~N}-7$ & 9961 & 91 & & & & \\
\hline $16 \mathrm{~N}-10$ & & & 7542 & 132 & 9814 & 42 \\
\hline $16 \mathrm{~N}-11$ & & & 7541 & 132 & 9813 & 42 \\
\hline
\end{tabular}

Theorem 4.24 For the numbers from groups 1 and $2, \delta(N)=\delta(24 N-5)-2$.

Proof $\quad N \stackrel{\times 12-2}{\longrightarrow} \underbrace{12 N-2}_{\delta+1} \stackrel{A^{-1}}{\longrightarrow} \underbrace{24 N-5}_{(\delta+1)+1}$.

Theorem 4.25 For the numbers from groups 1,2 , and $3, \delta(N)=\delta(24 N-7)-2$.

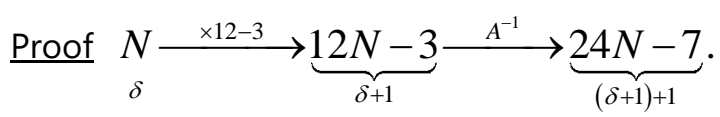

Theorem 4.26 If $\mathrm{N}$ is divisible by 4 , then $\delta(N)=\delta(24 N-11)-2$.

Proof $\quad N \stackrel{\times 12-5}{\longrightarrow} \underbrace{12 N-5}_{\delta+1} \stackrel{A^{-1}}{\longrightarrow} \underbrace{24 N-11}_{(\delta+1)+1}$.

Theorem 4.27 If $\mathrm{N}$ is even, then $\delta(N)=\delta(24 N-15)-2$. 
Proof $N \underset{\delta}{\stackrel{\times 12-7}{\longrightarrow}} \underbrace{2 N-7}_{\delta+1} \stackrel{A^{-1}}{\longrightarrow} \underbrace{24 N-15}_{(\delta+1)+1}$.

Example 4.9

\begin{tabular}{|l|l|l|l|l|l|l|}
\hline & Group 1 & index & Group 4 & index & Group 5 & index \\
\hline $\mathrm{N}$ & 412 & 133 & 376 & 45 & 406 & 27 \\
\hline $24 \mathrm{~N}-5$ & 9883 & 135 & & & & \\
\hline $24 \mathrm{~N}-7$ & 9881 & 135 & & & & \\
\hline $24 \mathrm{~N}-11$ & 9877 & 135 & 9013 & 47 & & \\
\hline $24 \mathrm{~N}-15$ & 9873 & 135 & 9009 & 47 & 9729 & 29 \\
\hline
\end{tabular}

Theorem 4.28 For the numbers from groups 1,2, and 3,

$$
\delta(N)=\delta(32 N-10)-5=\delta(32 N-11)-5 .
$$

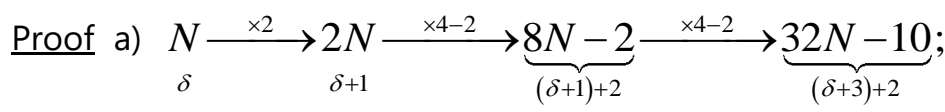

b) $N \stackrel{\times 2}{\longrightarrow} 2 N \stackrel{\times 4-2}{\longrightarrow} \underbrace{8 N-2}_{\delta+1} \stackrel{\times 4-3}{\longrightarrow} \underbrace{32 N-11}_{(\delta+3)+2}$.

Theorem 4.29 For the numbers from groups 1,2 , and $3, \delta(N)=\delta(32 N-15)-5$.

Proof a) $N \stackrel{\times 16-7}{\longrightarrow} \underbrace{16 N-7}_{\delta+4} \stackrel{A^{-1}}{\longrightarrow} \underbrace{32 N-15}_{(\delta+4)+1}$.

Theorem 4.30 If $\mathrm{N}$ is even, then $\delta(N)=\delta(32 N-21)-5=\delta(32 N-23)-5$.

Proof a) $N \stackrel{\times 16-10}{\longrightarrow} \underbrace{16 N-10}_{\delta+4} \stackrel{A^{-1}}{\longrightarrow} \underbrace{32 N-21}_{(\delta+4)+1}$;

b) $N \stackrel{\times 16-11}{\longrightarrow} \underbrace{16 N-11}_{\delta+4} \stackrel{A^{-1}}{\longrightarrow} \underbrace{32 N-23}_{(\delta+4)+1}$.

Example 4.10

\begin{tabular}{|l|l|l|l|l|l|l|}
\hline & Group 2 & index & Group 3 & index & Group 5 & index \\
\hline $\mathrm{N}$ & 282 & 42 & 275 & 91 & 278 & 16 \\
\hline $32 \mathrm{~N}-15$ & 9009 & 47 & 8785 & 96 & & \\
\hline
\end{tabular}




\begin{tabular}{|l|l|l|l|l|l|l|}
\hline $32 \mathrm{~N}-21$ & 9003 & 47 & & & 8875 & 21 \\
\hline $32 \mathrm{~N}-23$ & 9001 & 47 & & & 8873 & 21 \\
\hline
\end{tabular}

Fundamental Theorem.

We will try to prove that any natural number $\mathrm{N}$ is convergent to the number 2 (Ref.3,4).

More precisely, we will show that using Theorems proved in $4^{\circ}$, any natural number can be reduced to a smaller number. As we mentioned in $3^{\circ}$, we need to consider numbers that are divisible by 4 .

For each natural number $\mathrm{N}$, we will construct the sequence of increasing numbers (not consecutive) having the same index as the original number $\mathrm{N}$ (Ref.5).

Proposition 5.1 If $\mathrm{N}$ is odd, $N \geq 3$, then $\delta(4 N+2)=\delta(4 N+1)$.

Proof $4 N+2 \stackrel{B}{\longrightarrow} 6 N+3 \stackrel{A}{\longrightarrow} 3 N+2 \stackrel{A}{\longrightarrow} \frac{3 N+3}{2} ;$

$$
4 N+1 \stackrel{A}{\longrightarrow} 2 N+1 \stackrel{A}{\longrightarrow} N+1 \stackrel{B}{\longrightarrow} \frac{(N+1) \cdot 3}{2} .
$$

The last entries are the same after 4 steps.

Here are some examples of the pair of numbers with equal index:

\section{Example 5.1}

\begin{tabular}{|l|l|l|l|l|l|l|l|l|}
\hline $\mathrm{N}$ & 21 & index & 739 & index & 1873 & index & 2391 & index \\
\hline $4 \mathrm{~N}+1$ & 85 & 9 & 2957 & 22 & 7493 & 88 & 9565 & 122 \\
\hline $4 \mathrm{~N}+2$ & 86 & 9 & 2958 & 22 & 7494 & 88 & 9566 & 122 \\
\hline
\end{tabular}

Theorem $5.1 \delta\left[2^{p} \cdot(\right.$ odd number $\left.)+1\right]=\delta\left[2^{p} \cdot(\right.$ odd number $\left.)+1+2^{p-2}\right], \mathrm{p} \geq 2$ ，

$($ odd number $)=3,5,7, \ldots$.

Proof. The number in the left side $2^{p} \cdot($ odd number $)+1 \stackrel{A^{p-2}}{\longrightarrow} 2^{2} \cdot($ odd number $)+1$;

the number on the right side $2^{p} \cdot($ odd number $)+1+2^{p-2} \stackrel{A^{p-2}}{\longrightarrow} 2^{2} \cdot($ odd number $)+1+2^{0}$,

and by Proposition 5.1, $\delta[4 \cdot($ odd number $)+1]=\delta[4 \cdot($ odd number $)+2]$. 


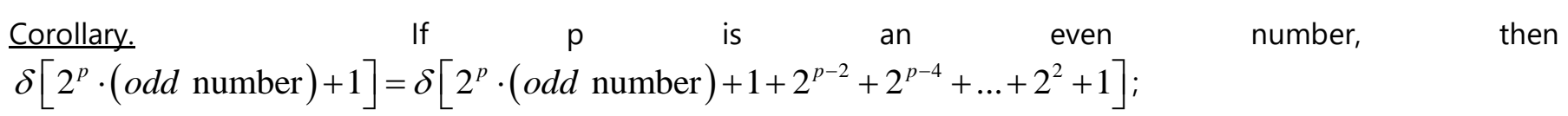
and if $p$ is an odd number, then $\delta\left[2^{p} \cdot(\right.$ odd number $\left.)+1\right]=\delta\left[2^{p} \cdot(\right.$ odd number $\left.)+1+2^{p-2}+2^{p-4}+\ldots+2^{3}+2\right]$.

Proof. The right side in Theorem 5.1 is

$\delta\left[2^{p}(\right.$ odd number $\left.)+1+2^{p-2}\right]=\delta\left\{2^{p-2}[4(\right.$ odd number $\left.)+1]+1\right\}$, and by applying

Theorem 5.1 again, we get $\delta\left[2^{p}(\right.$ odd number $\left.)+1+2^{p-2}+2^{p-4}\right]$ and continue by induction, we get desire conclusion.

Definition. Define the following operations:

$$
P(N)=6 N-3, Q(N)=6 N-1, R\left[2^{p}(\text { odd number })+1\right]=2^{p}(\text { odd number })+1+2^{p-2} .
$$

Now we will use those operations to construct for the natural number $N$ corresponding sequence of increasing numbers having the same index.

Example 5.2 For $\mathrm{N}=180$, using operations $P, Q$, and $R$, we get the sequence

$$
\begin{aligned}
& 180 \stackrel{P}{\longrightarrow} 1077\left(2^{2} \cdot 269+1\right) \stackrel{+2^{0}}{\longrightarrow} 1078 \stackrel{P}{\longrightarrow} 6465\left(2^{6} \cdot 101+1\right) \stackrel{+2^{4}+2^{2}+2^{0}}{\longrightarrow} 6486 \\
& \stackrel{P}{\longrightarrow} 38,913\left(2^{11} \cdot 19+1\right) \stackrel{+2^{9}+2^{7}+2^{5}+2^{3}+2^{1}}{\longrightarrow} 39595 \stackrel{Q}{\longrightarrow} 237,569\left(2^{13} \cdot 29+1\right) \\
& \stackrel{+2^{11}+2^{9}+2^{7}+2^{5}+2^{3}+2^{1}}{\longrightarrow} 240,299 \stackrel{Q}{\longrightarrow} 1,441,793\left(2^{17} \cdot 11+1\right) \stackrel{+2^{15}+2^{13}+2^{11}+2^{9}+2^{7}+2^{5}+2^{3}+2^{1}}{\longrightarrow} \\
& 1,485,483 \stackrel{Q}{\longrightarrow} 8,912,897\left(2^{19} \cdot 17+1\right) \stackrel{+2^{17}+2^{15}+2^{13}+2^{11}+2^{9}+2^{7}+2^{5}+2^{3}+2^{1}}{\longrightarrow} 9,087,659 \\
& \stackrel{Q}{\longrightarrow} 54,525,953\left(2^{22} \cdot 13+1\right) \stackrel{+2^{20}+2^{18}+2^{16}+2^{14}+2^{12}+2^{10}+2^{8}+2^{6}+2^{4}+2^{2}+2^{0}}{\longrightarrow} 55,924,054 \\
& \stackrel{P}{\longrightarrow} 335,544,321\left(2^{26} \cdot 5+1\right) \stackrel{+2^{24}+2^{22}+2^{20}+2^{18}+2^{16}+2^{14}+2^{12}+2^{10}+2^{8}+2^{6}+2^{4}+2^{2}+2^{0}}{\longrightarrow} 357,913,942 \\
& \stackrel{P}{\longrightarrow} 2,147,483,649=2^{31}+1 .
\end{aligned}
$$

Therefore, $\delta(180)=31$.

By the regular way, using operations $A$ and $B$, we have:

$$
\begin{aligned}
& 180 \stackrel{B}{\longrightarrow} 270 \stackrel{B}{\longrightarrow} 405 \stackrel{A}{\longrightarrow} 203 \stackrel{A}{\longrightarrow} 102 \stackrel{B}{\longrightarrow} 153 \stackrel{A}{\longrightarrow} 77 \stackrel{A}{\longrightarrow} 39 \stackrel{A}{\longrightarrow} 20 \stackrel{B}{\longrightarrow} 30 \\
& \stackrel{B}{\longrightarrow} 45 \stackrel{A}{\longrightarrow} 23 \stackrel{A}{\longrightarrow} 12 \stackrel{B}{\longrightarrow} 18 \stackrel{B}{\longrightarrow} 27 \stackrel{A}{\longrightarrow} 14 \stackrel{B}{\longrightarrow} 21 \stackrel{A}{\longrightarrow} 11 \stackrel{A}{\longrightarrow} 6 \stackrel{B}{\longrightarrow} 9
\end{aligned}
$$


$\stackrel{A}{\longrightarrow} 5 \stackrel{A}{\longrightarrow} 3 \stackrel{A}{\longrightarrow} 2$.

For the original Collatz Sequence for $N=180-1=179$, we have

$$
\begin{aligned}
& 179 \rightarrow 538 \rightarrow 269 \rightarrow 808 \rightarrow 404 \rightarrow 202 \rightarrow 101 \rightarrow 304 \rightarrow 152 \rightarrow 76 \rightarrow 38 \rightarrow 19 \rightarrow 58 \rightarrow 29 \\
& \rightarrow 88 \rightarrow 44 \rightarrow 22 \rightarrow 11 \rightarrow 34 \rightarrow 17 \rightarrow 52 \rightarrow 26 \rightarrow 13 \rightarrow 40 \rightarrow 20 \rightarrow 10 \rightarrow 5 \rightarrow 16 \rightarrow 8 \rightarrow 4 \\
& \rightarrow 2 \rightarrow 1 .
\end{aligned}
$$

Let us consider the increasing numbers in our sequence (5.1):

$1077=2^{2} \cdot 269+1 \stackrel{A^{2}}{\longrightarrow} 2^{0} \cdot 269+1=270 ;$

$1078 \stackrel{B}{\longrightarrow} 1617=2^{2} \cdot 404+1 \stackrel{A^{2}}{\longrightarrow} 2^{0} \cdot 404+1=405 ;$

$6465=2^{6} \cdot 101+1 \stackrel{A^{6}}{\longrightarrow} 2^{0} \cdot 101+1=102 ;$

$6486 \stackrel{B}{\longrightarrow} 9729=2^{6} \cdot 152+1 \stackrel{A^{6}}{\longrightarrow} 2^{0} \cdot 152+1=153 ;$

$38,913=2^{11} \cdot 19+1 \stackrel{A^{11}}{\longrightarrow} 2^{0} \cdot 19+1=20 ;$

$39,595 \stackrel{A}{\longrightarrow} 19,798 \stackrel{B}{\longrightarrow} 29,697=2^{10} \cdot 29+1 \stackrel{A^{10}}{\longrightarrow} 2^{0} \cdot 29+1=30$

$237,569=2^{13} \cdot 29+1 \stackrel{A^{13}}{\longrightarrow} 2^{0} \cdot 29+1=30$

$240,299 \stackrel{A}{\longrightarrow} 120,150 \stackrel{B}{\longrightarrow} 180,225=2^{12} \cdot 44+1 \stackrel{A^{12}}{\longrightarrow} 2^{0} \cdot 44+1=45 ;$

$1,441,793=2^{17} \cdot 11+1 \stackrel{A^{17}}{\longrightarrow} 2^{0} \cdot 11+1=12 ;$

$1,485,483 \stackrel{A}{\longrightarrow} 742,742 \stackrel{B}{\longrightarrow} 1,114,113=2^{16} \cdot 17+1 \stackrel{A^{16}}{\longrightarrow} 2^{0} \cdot 17+1=18 ;$

$8,912,897=2^{19} \cdot 17+1 \stackrel{A^{19}}{\longrightarrow} 2^{0} \cdot 17+1=18$

$9,087,659 \stackrel{A}{\longrightarrow} 4,543,830 \stackrel{B}{\longrightarrow} 6,815,745=2^{18} \cdot 26+1 \stackrel{A^{18}}{\longrightarrow} 2^{0} \cdot 26+1=27 ;$

$54,525,953=2^{22} \cdot 13+1 \stackrel{A^{22}}{\longrightarrow} 2^{0} \cdot 13+1=14$

$55,924,054 \stackrel{B}{\longrightarrow} 83,886,081=2^{22} \cdot 20+1 \stackrel{A^{22}}{\longrightarrow} 2^{0} \cdot 20+1=21 ;$

$335,544,321=2^{26} \cdot 5+1 \stackrel{A^{26}}{\longrightarrow} 2^{0} \cdot 5+1=6 ;$

$357,913,942 \stackrel{B}{\longrightarrow} 536,870,913=2^{26} \cdot 8+1 \stackrel{A^{26}}{\longrightarrow} 2^{0} \cdot 8+1=9 ;$ 
$2,147,483,649=2^{31}+1 \stackrel{A^{31}}{\longrightarrow} 2^{0}+1=2$.

We may see relations between sequences 5.1, 5.2, and 5.3. Sequence 5.3 converges to number 1 in 31 steps. Sequence 5.2 converges to number 2 in 31 steps (operation A gives one step, but operation B is equivalent to 2 steps). So, both sequences have the same index $\delta=31$, and we may see the relation between corresponding numbers of two sequences. Constructed sequence 5.1 contains increasing numbers, and all those numbers have the same index by Theorems 4.8, 4.10, and 5.1. Index of the last number of the sequence 5.1 is $\delta\left(2^{31}+1\right)=31$.

The number $2^{31}+1$ is the greatest natural number that has index 31. Further increasing this number changes index, $2^{31} \cdot 1+1 \stackrel{R}{\longrightarrow} 2^{31} \cdot 1+1+2^{29}=2^{29} \cdot 5+1 \stackrel{A^{29}}{\longrightarrow} 2^{0} \cdot 5+1=6$, therefore, $\delta\left(2^{31}+1+2^{29}\right)=29+\delta(6)=29+5=34$. That's why in the Theorem 5.1, (odd number) should be greater than

1. By the formulas 5.4 we recognize, that the increasing numbers in the constructed sequence 5.1 are related to corresponding numbers of the sequence 5.2. Collecting information in $5^{\circ}$, we may claim

Theorem 5.2 (Fundamental). For any natural number $N>2$, we may construct the sequence, using operations $P$, $Q, R$, which satisfy the following conditions:

a) sequence is increasing and bounded,

b) all numbers in the sequence have the same index,

c) the biggest number in the sequence is $2^{n}+1$, where $n=\delta(N)$.

\section{Conclusions:}

This proves the Collatz Conjecture for five out of six classes.

Funding Statement: Self-funded

Acknowledgment: To my wife, Tatiana

\section{References:}

1. Lagarias, Jeffrey C. (1985). "The $3 x+1$ problem and its generalizations". The American Mathematical Monthly. 92 (1): 3-23. JSTOR 2322189.

2. Nikolaychuk, Anatoliy (2018). "Supplement to The Collatz Conjecture." Journal Of Advances in Mathematics, Vol. 15, pp. 8120-8132. ISSN:2347 - 1921.

3. Guy, Richard K. (2004). "'E17: Permutation Sequences"'. Unsolved problems in number theory (3rd ed.). Springer-Verlag. pp. 336-7. ISBN $\underline{\mathrm{Zbl}} \underline{1058.11001 .}$

4. Guy, R. K. (1983). "Don't try to solve these problems." Amer. Math. Monthly. 90: 3541. DOI:10.2307/2975688. JSTOR 2975688. By this Erdos means that there aren't powerful tools for manipulating such objects.

5. Roosendaal, Eric. "3x+1 Delay Records". Retrieved 30 June 2017. (Note: "Delay records" are total stopping time records.) 